\title{
Cosmological implications of the transition from the false vacuum to the true vacuum state
}

\author{
Aleksander Stachowski ${ }^{1, a}$, Marek Szydłowski ${ }^{1,2, b}$, Krzysztof Urbanowski $^{3, c}$ \\ ${ }^{1}$ Astronomical Observatory, Jagiellonian University, Orla 171, 30-244 Kraków, Poland \\ ${ }^{2}$ Mark Kac Complex Systems Research Centre, Jagiellonian University, Łojasiewicza 11, 30-348 Kraków, Poland \\ ${ }^{3}$ Institute of Physics, University of Zielona Góra, Prof. Z. Szafrana 4a, 65-516 Zielona Gora, Poland
}

Received: 19 April 2017 / Accepted: 19 May 2017 / Published online: 30 May 2017

(C) The Author(s) 2017. This article is an open access publication

\begin{abstract}
We study cosmology with running dark energy. The energy density of dark energy is obtained from the quantum process of transition from the false vacuum state to the true vacuum state. We use the Breit-Wigner energy distribution function to model the quantum unstable systems and obtain the energy density of the dark energy parametrization $\rho_{\text {de }}(t)$. We also use Krauss and Dent's idea linking properties of the quantum mechanical decay of unstable states with the properties of the observed Universe. In the cosmological model with this parametrization there is an energy transfer between dark matter and dark energy. The intensity of this process, measured by a parameter $\alpha$, distinguishes two scenarios. As the Universe starts from the false vacuum state, for the small value of $\alpha(0<\alpha<0.4)$ it goes through an intermediate oscillatory (quantum) regime of the density of dark energy, while for $\alpha>0.4$ the density of the dark energy jumps down. In both cases the present value of the density of dark energy is reached. From a statistical analysis we find this model to be in good agreement with the astronomical data and practically indistinguishable from the $\Lambda \mathrm{CDM}$ model.
\end{abstract}

\section{Introduction}

The standard cosmological model ( $\Lambda \mathrm{CDM}$ model), which describes the Universe, is the one most favored by astronomical observations such as supernovae of type Ia or measurements of CMB. In the $\Lambda$ CDM model, the dark matter is treated as dust and dark energy has the form of the cosmological constant $\Lambda_{\text {bare }}$. We are looking for an alternative for the $\Lambda \mathrm{CDM}$ model by a modification of the dark energy term.

\footnotetext{
a e-mail: aleksander.stachowski@uj.edu.pl

b e-mail: marek.szydlowski@uj.edu.pl

c e-mail: K.Urbanowski@if.uz.zgora.pl
}

The standard cosmological model possesses the six parameters: the density of baryons $\Omega_{\mathrm{b}} h^{2}$, the density of cold dark matter $\Omega_{\mathrm{dm}} h^{2}$, the angular diameter of sound horizon at last scattering $\theta$, the optical depth due to the reionization $\tau_{\mathrm{R}}$, the slope of the primordial power spectrum of fluctuations $n_{\mathrm{s}}$, and the amplitude of the primordial power spectrum $A_{\mathrm{s}}$, where $h=H_{0}\left(100 \mathrm{~km} \mathrm{~s}^{-1} \mathrm{Mpc}^{-1}\right)$.

From the methodological point of view, the standard cosmological model plays the role of an effective theory, which very well describes properties of the current Universe without explaining the nature of two components of the model: the dark energy and the dark matter. The nature of both components of the Universe has been unknown up to now but we describe these in terms of some useful fiction, the cosmological constant and the cold dark matter, which is a kind of a dust perfect fluid.

In this paper we concentrate on the interpretation of dark energy in terms of running cosmological constant rather than in terms of the pure cosmological constant parameter $\left(\Lambda_{\text {bare }}\right.$ in our approach). It is a consequence of some problems with the interpretation of the pure cosmological constant, namely:

1. One cannot explain why the cosmological constant is not large.

2. One does not know why it is not just equal zero.

3. One cannot explain why energy densities of both dark energy and dark matter, expressed in terms of dimensionless density parameters, are comparable in the current epoch (cosmic coincidence problem).

In our proposition of the explanation of these problems with the cosmological constant parameter, we base our ideas on the theories of the cosmological constant in which the vacuum energy is fixed by the fundamental theory [1]. Extending the $\Lambda \mathrm{CDM}$ model beyond the classical regime, we apply quantum mechanics as a fundamental theory, which deter- 
mines cosmological parameters and we explain how cosmological parameters vary during the cosmic evolution.

The cosmological constant is the source of two problems in modern cosmology. The first problem is the cosmological constant problem, which is consequence of the interpretation of dark energy as a vacuum energy. The observed present value of the cosmological constant is 120 orders of magnitude smaller than we expect from quantum physics. The second problem is the coincidence problem. If we assume that the dark energy is always constant, then the $\Lambda \mathrm{CDM}$ model cannot explain why the cosmological constant has the same order of magnitude as the density of matter today. If the model belongs to the class of running dark energy cosmologies then the first problem of cosmological constant can be solved.

This question seems to be crucial in contemporary physics because its solution would certainly mean a very crucial step forward in our attempts to understand physics from the boundary of particle physics and cosmology. A discussion as regards the cosmological constant problem can be found in Refs. [1-16].

In our model, the influence of running dark energy densities of both visible and invisible matter is very small. Thus we share Weinberg's opinion, according to which looking for a solution of the coincidence problem, we should consider the anthropic principle. According to Weinberg's argument, no observers at all should be in the Universe if the cosmological constant was even three orders of magnitude larger than it is now.

Coleman et al. [17-19] discussed the instability of a physical system, which is not at an absolute energy minimum, and which is separated from the minimum by an effective potential barrier. They showed that if the early Universe is too cold to activate the energy transition to the minimum energy state, then a quantum decay, from the false vacuum to the true vacuum, is still possible through a barrier penetration via macroscopic quantum tunneling.

The discovery of the Higgs-like resonance at 125-126 $\mathrm{GeV}$ [20-23] caused a discussion as regards the instability of the false vacuum. If we assume that the Standard Model well describes the evolution of the Universe up to the Planck epoch, then a Higgs mass $m_{\mathrm{h}}<126 \mathrm{GeV}$ causes the electroweak vacuum to be in a metastable state [21]. In consequence the instability of the Higgs vacuum should be considered in the cosmological models of the early time Universe.

The idea that properties of the quantum mechanical decay process of metastable states can help to understand the properties of the observed Universe was formulated in [24-26]. It is because the decay of the false vacuum is a quantum decay process [17-19]. This means that the state vector corresponding to the false vacuum is a quantum unstable (or metastable) state. Therefore all general properties of quantum unstable systems must also occur in the case of such a quantum unsta- ble state as the false vacuum and, as a consequence, models of quantum unstable systems can be used to analyze properties of the systems of which the time evolution starts from the false vacuum state. Note that Landim and Abdalla built a model of metastable dark energy, in which the observed vacuum energy is the value of the scalar potential at the false vacuum [27].

In this paper, we assume the Breit-Wigner energy distribution function, which is very often used to model unstable quantum systems, as a model of the process of the energy transition from the false vacuum to the true vacuum. In consequence the parametrization of the dark energy is given by formula

$\rho_{\mathrm{de}}=E_{0}+E_{\mathrm{R}} \frac{\alpha}{1-\alpha} \Re\left(\frac{J(t)}{I(t)}\right)$,

where $\alpha$ and $E_{\mathrm{R}}$ are model parameters describing the variation from the standard cosmological model. The values of the parameter $\alpha$ belong to interval $\langle 0,1)$. Note that if the parameter $\alpha$ or $E_{\mathrm{R}}$ is equal to zero, then the model is equivalent to the $\Lambda \mathrm{CDM}$ model.

Let $\Lambda_{\text {bare }}=E_{0}-E_{\mathrm{R}}$; then Eq. (1) can be rewritten in the equivalent form

$\rho_{\text {de }}=\Lambda_{\text {bare }}+E_{\mathrm{R}}\left[1+\frac{\alpha}{1-\alpha} \Re\left(\frac{J(t)}{I(t)}\right)\right]$.

Here the units $8 \pi G=c=1$ are used.

The functions $J(t)$ and $I(t)$ are defined by the following expressions:

$J(t)=\int_{-\frac{1-\alpha}{\alpha}}^{\infty} \frac{\eta}{\eta^{2}+\frac{1}{4}} e^{-i \eta \tau} \mathrm{d} \eta$,

$I(t)=\int_{-\frac{1-\alpha}{\alpha}}^{\infty} \frac{1}{\eta^{2}+\frac{1}{4}} e^{-i \eta \tau} \mathrm{d} \eta$.

The integrals $J(t)$ and $I(t)$ can be expressed by the exact solutions of these integrals. The formula for $J(t)$ is the following expression:

$$
\begin{aligned}
J(\tau)= & \frac{1}{2} e^{-\tau / 2}\left(-2 i \pi+e^{\tau} \mathrm{E}_{1}\left(\left[\frac{1}{2}-\frac{i(1-\alpha)}{\alpha}\right] \tau\right)\right. \\
& \left.+\mathrm{E}_{1}\left(\left[-\frac{1}{2}-\frac{i(1-\alpha)}{\alpha}\right] \tau\right)\right)
\end{aligned}
$$

and $I(t)$ is expressed by

$$
\begin{aligned}
I(\tau)= & 2 \pi e^{-\tau / 2}\left(1+\frac{i}{2 \pi}\left(-e^{\tau} \mathrm{E}_{1}\left(\left[\frac{1}{2}-\frac{i(1-\alpha)}{\alpha}\right] \tau\right)\right.\right. \\
& \left.\left.+\mathrm{E}_{1}\left(\left[-\frac{1}{2}-\frac{i(1-\alpha)}{\alpha}\right] \tau\right)\right)\right),
\end{aligned}
$$

where $\tau=\frac{\alpha\left(E_{0}-\Lambda_{\text {bare }}\right)}{\hbar(1-\alpha)} V_{0} t$ and $V_{0}$ is the volume of the Universe in the Planck epoch. In this paper we assume that 
$V_{0}=1$. The function $E_{1}(z)$ is called the exponential integral and is defined by the formula: $E_{1}(z)=\int_{z}^{\infty} \frac{e^{-x}}{x} \mathrm{~d} x$ (see $[28,29])$.

\section{Preliminaries: unstable states}

As mentioned in Sect. 1 we will use the parametrization of the dark energy transition from the false vacuum state to the true vacuum state following from the quantum properties of such a process. This process is a quantum decay process, so we need quantities characterizing decay processes of quantum unstable systems. The main information as regards properties of quantum unstable systems is contained in their decay law, that is, in their survival probability. So if one knows that the system is in the initial unstable state $|\phi\rangle \in \mathcal{H}(\mathcal{H}$ is the Hilbert space of states of the considered system), which was prepared at the initial instant $t_{0}=0$, then one can calculate its survival probability (the decay law), $\mathcal{P}(t)$, of the unstable state $|\phi\rangle$ decaying in vacuum, which equals

$\mathcal{P}(t)=|A(t)|^{2}$,

where $A(t)$ is the probability amplitude of finding the system at time $t$ in the rest frame $\mathcal{O}_{0}$ in the initial unstable state $|\phi\rangle$,

$A(t)=\langle\phi \mid \phi(t)\rangle$,

and $|\phi(t)\rangle$ is the solution of the Schrödinger equation for the initial condition $|\phi(0)\rangle=|\phi\rangle$, which has the following form:

$i \hbar \frac{\partial}{\partial t}|\phi(t)\rangle=\mathfrak{H}|\phi(t)\rangle$

Here $|\phi\rangle,|\phi(t)\rangle \in \mathcal{H}$, and $\mathfrak{H}$ is the total self-adjoint Hamiltonian for the system considered. The spectrum of $\mathfrak{H}$ is assumed to be bounded from below, $E_{\min }>-\infty$ is the lower bound of the spectrum $\sigma_{c}(\mathfrak{H})=\left[E_{\min },+\infty\right)$ of $\left.\mathfrak{H}\right)$. Using the basis in $\mathcal{H}$ built from normalized eigenvectors $|E\rangle, \quad E \in \sigma_{c}(\mathfrak{H})$ of $\mathfrak{H}$ and expanding $|\phi\rangle$ in terms of these eigenvectors one can express the amplitude $A(t)$ as the following Fourier integral:

$A(t) \equiv \int_{E_{\min }}^{\infty} \omega(E) e^{-\frac{i}{\hbar} E t} \mathrm{~d} E$,

where $\omega(E)>0$ (see [30-32]).

So the amplitude $A(t)$ and, thus, the decay law $\mathcal{P}(t)$ of the unstable state $|\phi\rangle$ are completely determined by the density of the energy distribution $\omega(E)$ for the system in this state [30,31]; see also [32-39] (this approach is also applicable in Quantum Field Theory models [40,41]).

Note that in fact the amplitude $A(t)$ contains information as regards the decay law $\mathcal{P}_{\phi}(t)$ of the state $|\phi\rangle$, that is, as regards the decay rate $\Gamma_{\phi}^{0}$ of this state, as well as the energy $E_{\phi}^{0}$ of the system in this state. This information can be extracted from $A(t)$. It can be done using the rigorous equation governing the time evolution in the subspace of unstable states, $\mathcal{H}_{\|} \ni|\phi\rangle_{\|} \equiv|\phi\rangle$. Such an equation follows from the Schrödinger equation (9) for the total state space $\mathcal{H}$.

The use of the Schrödinger equation (9) allows one to find that within the problem considered

$i \hbar \frac{\partial}{\partial t}\langle\phi \mid \phi(t)\rangle=\langle\phi|\mathfrak{H}| \phi(t)\rangle$.

This relation leads to the conclusion that the amplitude $A(t)$ satisfies the following equation:

$i \hbar \frac{\partial A(t)}{\partial t}=h(t) A(t)$

where

$h(t)=\frac{\langle\phi|\mathfrak{H}| \phi(t)\rangle}{A(t)}$

and $h(t)$ is the effective Hamiltonian governing the time evolution in the subspace of unstable states $\mathcal{H}_{\|}=\mathbb{P} \mathcal{H}$, where $\mathbb{P}=|\phi\rangle\langle\phi|$ (see [42] and also [43,44] and the references therein). The subspace $\mathcal{H} \ominus \mathcal{H}_{\|}=\mathcal{H}_{\perp} \equiv \mathbb{Q} \mathcal{H}$ is the subspace of decay products. Here $\mathbb{Q}=\mathbb{I}-\mathbb{P}$. We have the following equivalent formula for $h(t)$ [42-44]:

$h(t) \equiv \frac{i \hbar}{A(t)} \frac{\partial A(t)}{\partial t}$.

One meets the effective Hamiltonian $h(t)$ when one starts with the Schrödinger equation for the total state space $\mathcal{H}$ and looks for the rigorous evolution equation for a distinguished subspace of states $\mathcal{H}_{\|} \subset \mathcal{H}[39,42]$. In general $h(t)$ is a complex function of time and in the case of $\mathcal{H}_{\|}$is dimension two or more the effective Hamiltonian governing the time evolution in such a subspace it is a non-hermitian matrix $H_{\|}$ or non-hermitian operator. We have

$h(t)=E_{\phi}(t)-\frac{i}{2} \Gamma_{\phi}(t)$

and

$E_{\phi}(t)=\Re[h(t)], \quad \Gamma_{\phi}(t)=-2 \mathfrak{s}[h(t)]$,

are the instantaneous energy (mass) $E_{\phi}(t)$ and the instantaneous decay rate, $\Gamma_{\phi}(t)$ [42-44]. Here $\Re(z)$ and $\Im(z)$ denote the real and imaginary parts of $z$, respectively. Equations (12), (14) and (16) are convenient when the density $\omega(E)$ is given and one wants to find the instantaneous energy $E_{\phi}(t)$ and decay rate $\Gamma_{\phi}(t)$ : Inserting $\omega(E)$ into (10) one obtains the amplitude $A(t)$ and then using (14) one finds $h(t)$ and thus 
$E_{\phi}(t)$ and $\Gamma_{\phi}(t)$. The simplest choice is to take $\omega(E)$ in the Breit-Wigner form,

$\omega(E) \equiv \omega_{\mathrm{BW}}(E) \stackrel{\text { def }}{=} \frac{N}{2 \pi} \frac{\Gamma_{0} \Theta\left(E-E_{\min }\right)}{\left(E-E_{0}\right)^{2}+\left(\frac{\Gamma_{0}}{2}\right)^{2}}$,

where $N$ is a normalization constant and $\Theta(E)=1$ for $E \geq 0$ and $\Theta(E)=0$ for $E<0$. The parameters $E_{0}$ and $\Gamma_{0}$ correspond to the energy of the system in the unstable state and its decay rate at the exponential (or canonical) regime of the decay process. $E_{\min }$ is the minimal (the lowest) energy of the system. Inserting $\omega_{B W}(E)$ into Eq. (10) for the amplitude $A(t)$ after some algebra one finds that

$A(t)=\frac{N}{2 \pi} e^{-\frac{i}{\hbar} E_{0} t} I_{\beta}\left(\frac{\Gamma_{0} t}{\hbar}\right)$,

where

$I_{\beta}(\tau) \stackrel{\text { def }}{=} \int_{-\beta}^{\infty} \frac{1}{\eta^{2}+\frac{1}{4}} e^{-i \eta \tau} \mathrm{d} \eta$.

Here $\tau=\frac{\Gamma_{0} t}{\hbar} \equiv \frac{t}{\tau_{0}}, \tau_{0}$ is the lifetime and $\beta=\frac{E_{0}-E_{\min }}{\Gamma_{0}}$. The integral $I_{\beta}(t)$ can be expressed in terms of special functions as follows:

$$
\begin{aligned}
I_{\beta}(\tau)= & 2 \pi e^{-\frac{\tau}{2}}+i\left\{e^{-\frac{\tau}{2}} E_{1}\left(-i\left(\beta-\frac{i}{2}\right) \tau\right)\right. \\
& \left.-e^{+\frac{\tau}{2}} E_{1}\left(-i\left(\beta+\frac{i}{2}\right) \tau\right)\right\},
\end{aligned}
$$

where $E_{1}(z)$ denotes the integral-exponential function defined according to [28,29] ( $z$ is a complex number).

Next using this $A(t)$ given by Eqs. (18), (19) and (14), defining the effective Hamiltonian $h_{\phi}(t)$, one finds that within the Breit-Wigner model considered

$h(t)=i \hbar \frac{1}{A(t)} \frac{\partial A(t)}{\partial t}=E_{0}+\Gamma_{0} \frac{J_{\beta}\left(\frac{\Gamma_{0} t}{\hbar}\right)}{I_{\beta}\left(\frac{\Gamma_{0} t}{\hbar}\right)}$,

where

$J_{\beta}(\tau)=\int_{-\beta}^{\infty} \frac{x}{x^{2}+\frac{1}{4}} e^{-i x \tau} \mathrm{d} x$.

It is important to be aware of the following problem: Namely from the definition of $J_{\beta}(\tau)$ one can conclude that $J_{\beta}(0)$ is undefined $\left(\lim _{\tau \rightarrow 0} J_{\beta}(\tau)=\infty\right)$. This is because within the model defined by the Breit-Wigner distribution of the energy density, $\omega_{B W}(E)$, the expectation value of $\mathfrak{H}$, that is, $\langle\phi|\mathfrak{H}| \phi\rangle$, is not finite. So all the considerations based on the use of $J_{\beta}(\tau)$ are valid only for $\tau>0$.

Note that simply

$J_{\beta}(\tau) \equiv i \frac{\partial I_{\beta}(\tau)}{\partial \tau}$, which allows one to find analytical form of $J_{\beta}(\tau)$ having such a form for $I_{\beta}(\tau)$.

We need to know the energy of the system in the unstable state $|\phi\rangle$ considered. The instantaneous energy $E_{\phi}(t)$ of the system in the unstable state $|\phi\rangle$ is given by Eq. (16). So within the Breit-Wigner model one finds that

$E_{\phi}(t)=E_{0}+\Gamma_{0} \Re\left[\frac{J_{\beta}\left(\frac{\Gamma_{0} t}{\hbar}\right)}{I_{\beta}\left(\frac{\Gamma_{0} t}{\hbar}\right)}\right]$

or, equivalently,

$\kappa(t) \stackrel{\text { def }}{=} \frac{E_{\phi}(t)-E_{\min }}{E_{0}-E_{\min }}=1+\frac{1}{\beta} \Re\left[\frac{J_{\beta}\left(\frac{\Gamma_{0} t}{\hbar}\right)}{I_{\beta}\left(\frac{\Gamma_{0} t}{\hbar}\right)}\right]$.

(This relation, i.e. $\kappa(t)$, was studied, for example in $[45,46]$.)

It is relatively simple to find asymptotic expressions $I_{\beta} \tau$ and $J_{\beta}(\tau)$ for $\tau \rightarrow \infty$ directly from (19) and (22), using, e.g., the method of integration by parts. We have, for $\tau \rightarrow \infty$,

$$
\begin{aligned}
I_{\beta}(\tau) \simeq & \frac{i}{\tau} \frac{e^{i \beta \tau}}{\beta^{2}+\frac{1}{4}}\left\{-1+\frac{2 \beta}{\beta^{2}+\frac{1}{4}} \frac{i}{\tau}\right. \\
& \left.+\left[\frac{2}{\beta^{2}+\frac{1}{4}}-\frac{8 \beta^{2}}{\left(\beta^{2}+\frac{1}{4}\right)^{2}}\right]\left(\frac{i}{\tau}\right)^{2}+\cdots\right\}
\end{aligned}
$$

and

$$
\begin{aligned}
J_{\beta}(\tau) \simeq & \frac{i}{\tau} \frac{e^{i \beta \tau}}{\beta^{2}+\frac{1}{4}}\left\{\beta+\left[1-\frac{2 \beta^{2}}{\beta^{2}+\frac{1}{4}}\right] \frac{i}{\tau}\right. \\
& \left.+\frac{\beta}{\beta^{2}+\frac{1}{4}}\left[\frac{8 \beta^{2}}{\beta^{2}+\frac{1}{4}}-6\right]\left(\frac{i}{\tau}\right)^{2}+\cdots\right\} .
\end{aligned}
$$

These two last asymptotic expressions allow one to find for $\tau \rightarrow \infty$ the asymptotic form of the ratio $\frac{J_{\beta}(\tau)}{I_{\beta}(\tau)}$ used in Eqs. (21), (24) and (25); it has a much simpler form than the asymptotic expansions for $I_{\beta}(\tau)$ and $J_{\beta}(\tau)$. One finds that, for $\tau \rightarrow \infty$,

$\frac{J_{\beta}(\tau)}{I_{\beta}(\tau)} \simeq-\beta-\frac{i}{\tau}-\frac{2 \beta}{\beta^{2}+\frac{1}{4}} \frac{1}{\tau^{2}}+\cdots$

Starting from this asymptotic expression and Eq. (24) or making use of the asymptotic expansion of $E_{1}(z)$ [29] and (20),

$E_{1}(z)_{|z| \rightarrow \infty} \sim \frac{e^{-z}}{z}\left(1-\frac{1}{z}+\frac{2}{z^{2}}-\cdots\right)$,

where $|\arg z|<\frac{3}{2} \pi$, one finds, e.g., that, for $t \rightarrow \infty$,

$E_{\phi}(t)_{t \rightarrow \infty} \simeq E_{\min }-2 \frac{E_{0}-E_{\min }}{\left|h_{\phi}^{0}-E_{\min }\right|^{2}}\left(\frac{\hbar}{t}\right)^{2}$, 
where $h_{\phi}^{0}=E_{0}-\frac{i}{2} \Gamma_{0}$. This last relation is valid for $t>$ $T$, where $T$ denotes the cross-over time, i.e. the time when exponential and late time inverse power law contributions to the survival amplitude begin to be comparable.

Some cosmological scenarios predict the possibility of decay of the Standard Model vacuum at an inflationary stage of the evolution of the Universe (see, e.g., [47] and also [48] and the references therein) or earlier. Of course this decaying Standard Model vacuum is described by the quantum state corresponding to a local minimum of the energy density, which is not the absolute minimum of the energy density of the system considered (see, e.g., Fig. 1). The scenario in which false vacuum may decay at the inflationary stage of the Universe corresponds with the hypothesis analyzed by Krauss and Dent [24,25]. Namely in the mentioned papers the hypothesis that some false vacuum regions do survive well up to the cross-over time $T$ or later was considered where $T$ is the same cross-over time as is considered within the theory of evolving in time quantum unstable systems. The fact that the decay of the false vacuum is a quantum decay process means that the state vector corresponding to the false vacuum is a quantum unstable (or metastable) state. Therefore all the general properties of quantum unstable systems must also occur in the case of such a quantum unstable state as the false vacuum. This applies in particular to such properties as late time deviations from the exponential decay law and properties of the energy $E_{0}^{\text {false }}(t)$ of the system in the quantum false vacuum state at late times $t>T$. In [49] it was pointed out that the energy of those false vacuum regions which survived up to $T$ and much later differs from $E_{0}^{\text {false }}$ [49].

So within the cosmological scenario in which the decay of a false vacuum is assumed the unstable state $|\phi\rangle$ corresponds to the false vacuum state: $|\phi\rangle=|0\rangle^{\text {false }}$. Then $|0\rangle^{\text {true }}$ is the true vacuum state, that is, the state corresponding to the true minimal energy. In such a case $E_{0} \rightarrow E_{0}^{\text {false }}$ is the energy of a state corresponding to the false vacuum measured at the canonical decay time (the exponential decay regime) and $E_{0}^{\text {true }}$ is the energy of true vacuum (i.e., the true ground state of the system), so $E_{0}^{\text {true }} \equiv E_{\min }$. The corresponding quantum mechanical process looks as shown in Fig. 1.

If one wants to generalize the above results, obtained on the basis of quantum mechanics, to quantum field theory one should take into account among others a volume factor so that survival probabilities per unit volume should be considered and similarly the energies and the decay rate, $E \mapsto \rho(E)=\frac{E}{V_{0}}, \Gamma_{0} \mapsto \gamma=\frac{\Gamma_{0}}{V_{0}}$, where $V_{0}=V\left(t_{0}\right)$ is the volume of the considered system at the initial instant $t_{0}$, when the time evolution starts. The volume $V_{0}$ is used in these considerations because the initial unstable state $|\phi\rangle \equiv|0\rangle^{\text {false }}$ at $t=t_{0}=0$ is expanded into eigenvectors $|E\rangle$ of $\mathfrak{H}$ at this initial instant $t_{0}$ (where $E \in \sigma_{c}(\mathfrak{H})$ ) and then this expansion is used to find the density of the energy distribution $\omega(E)$.

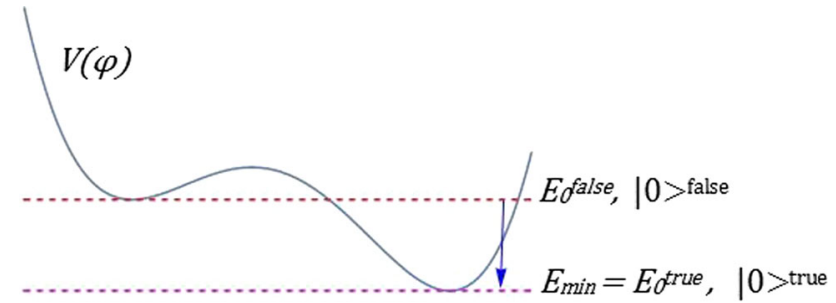

Fig. 1 Transition of the system from the false vacuum state $|0\rangle^{\text {false }}$ to the true ground state of the system, i.e. the true vacuum state $|0\rangle^{\text {true }}$. The states $|0\rangle^{\text {false }}$ and $|0\rangle^{\text {true }}$ correspond to the local minimum and to the true lowest minimum of the potential $V(\varphi)$ of the scalar field $\varphi$, respectively

It is easy to see that the mentioned changes $E \mapsto \frac{E}{V_{0}}$ and $\Gamma_{0} \mapsto \frac{\Gamma_{0}}{V_{0}}$ do not change the integrals $I_{\beta}(t)$ and $J_{\beta}(t)$ and Eq. (25). Similarly in such a situation the parameter $\beta=\frac{E_{0}-E_{\min }}{\Gamma_{0}}$ does not change. This means that Eqs. (24), (25), (30) can be replaced by the corresponding relations for the densities $\rho_{\text {de }}$ or $\Lambda$ (see, e.g., [45,51,52]). Within such an approach $E(t)$ corresponds to the running cosmological constant $\Lambda(t)$ and $E_{\min }$ to the $\Lambda_{\text {bare }}$. The parametrization used in next sections is based on Eqs. (24) and (25). The integrals (3), (4) introduced in Sect. 1 are obtained from (22) and (19) replacing $\beta$ by $\frac{1-\alpha}{\alpha}$. Similarly solutions (5) and (6) correspond to (20) and to the function $J_{\beta}(\tau)$ obtained from (20) using (23).

\section{Cosmological equations with

$$
\rho_{\mathrm{de}}=\Lambda_{\text {bare }}+E_{\mathrm{R}}\left[1+\frac{\alpha}{1-\alpha} \Re\left(\frac{J(t)}{I(t)}\right)\right]
$$

The cosmological model with the parametrization of the dark energy (1), belonging to the class of parametrizations proposed in [45] after putting $E_{R}=E_{0}-\Lambda_{\text {bare }}$, assumes the following form of $\rho_{\mathrm{de}}$ (we use units $8 \pi G=c=1$ ):

$\rho_{\mathrm{de}}=\Lambda_{\text {bare }}+E_{\mathrm{R}}\left[1+\frac{\alpha}{1-\alpha} \Re\left(\frac{J(t)}{I(t)}\right)\right]$.

It can be introduced as the covariant theory from the following action:

$S=\int \sqrt{-g}\left(R+\mathcal{L}_{\mathrm{m}}\right) \mathrm{d}^{4} x$,

where $R$ is the Ricci scalar, $\mathcal{L}_{\mathrm{m}}$ is the Lagrangian for the barotropic fluid and $g_{\mu \nu}$ is the metric tensor. We assume the signature of the metric tensor to be $(+,-,-,-)$ and, for simplicity, we assume that the constant curvature is zero (the flat model). The Ricci scalar for the Friedmann-LemaitreRobertson-Walker (FLRW) metric is represented by the following formula: 
$R=-6\left[\frac{\ddot{a}}{a}+\left(\frac{\dot{a}}{a}\right)^{2}\right]$

where a dot means differentiation with respect to the cosmological time $t$.

The Lagrangian for the barotropic fluid is expressed by the formula

$\mathcal{L}_{\mathrm{m}}=-\rho_{\mathrm{tot}}\left(1+\int \frac{p_{\mathrm{tot}}\left(\rho_{\mathrm{tot}}\right)}{\rho_{\mathrm{tot}}^{2}} \mathrm{~d} \rho_{\mathrm{tot}}\right)$,

where $\rho_{\text {tot }}$ is the total density of fluid and $p_{\text {tot }}\left(\rho_{\text {tot }}\right)$ is the total pressure of fluid [53]. We assume that this fluid consists of three components: the baryonic matter $\rho_{\mathrm{b}}$, the dark matter $\rho_{\mathrm{dm}}$ and the dark energy $\rho_{\mathrm{de}}$. We treat the baryonic matter and the dark matter like dust. In consequence the equations of state for them are the following: $p_{\mathrm{b}}\left(\rho_{\mathrm{b}}\right)=0$ and $p_{\mathrm{dm}}\left(\rho_{\mathrm{dm}}\right)=$ 0 . The equation of state for the dark energy is assumed in the form $p_{\mathrm{de}}\left(\rho_{\mathrm{de}}\right)=-\rho_{\mathrm{de}}$.

Of course, the total density is expressed by $\rho_{\text {tot }}=\rho_{\mathrm{b}}+$ $\rho_{\mathrm{dm}}+\rho_{\mathrm{de}}$ and the total pressure is expressed by $p_{\text {tot }}\left(\rho_{\text {tot }}\right)=$ $p_{\text {de }}\left(\rho_{\text {de }}\right)=-\rho_{\text {de }}$.

We can find the Einstein equations using the method of calculus of variations by variation of the action (32) by the metric $g_{\mu \nu}$. Then we get two equations: the Friedmann equation

$3 H^{2}=3 \frac{\dot{a}}{a}^{2}=\rho_{\mathrm{tot}}=\rho_{\mathrm{b}}+\rho_{\mathrm{dm}}+\rho_{\mathrm{de}}$,

where $H=\frac{\dot{a}}{a}$ is the Hubble function, and the acceleration equation

$\frac{\ddot{a}}{a}=-\frac{1}{6}\left(\rho_{\mathrm{tot}}+3 p_{\mathrm{tot}}\left(\rho_{\mathrm{tot}}\right)\right)=\rho_{\mathrm{b}}+\rho_{\mathrm{dm}}-2 \rho_{\mathrm{de}}$.

From Eqs. (35) and (36) we can get the conservation equation

$\dot{\rho}_{\text {tot }}=-3 H\left(\rho_{\text {tot }}+p_{\text {tot }}\left(\rho_{\text {tot }}\right)\right)$.

The above equation can be rewritten as

$\dot{\rho}_{\mathrm{m}}=-3 H \rho_{\mathrm{m}}-\dot{\rho}_{\mathrm{de}}$

where $\rho_{\mathrm{m}}=\rho_{\mathrm{b}}+\rho_{\mathrm{dm}}$.

Let $Q$ be the interaction between the dark matter and the dark energy. Then Eq. (38) is equivalent to the following equations:

$$
\begin{aligned}
\dot{\rho}_{\mathrm{b}} & =-3 H \rho_{\mathrm{b}}, \\
\dot{\rho}_{\mathrm{dm}} & =-3 H \rho_{\mathrm{dm}}+Q,
\end{aligned}
$$

and

$\dot{\rho}_{\mathrm{de}}=-Q$,

where the interaction $Q$ is defined by Eq. (41). The interaction between the dark matter and the dark energy can be interpreted as the energy transfer in the dark sector. If $Q>0$ then the energy flow is from the dark energy to the dark matter. If $Q<0$ then the energy flow is from the dark matter to the dark energy.

For the description of the evolution of the Universe it is necessary to use the Friedmann equation (35) and the conservation equation (38). These formulas can be rewritten in terms of dimensionless parameters. Let $\Omega_{\mathrm{m}}=\frac{\rho_{\mathrm{m}}}{3 H_{0}^{2}}$ and $\Omega_{\mathrm{de}}=\frac{\rho_{\mathrm{de}}}{3 H_{0}^{2}}$, where $H_{0}$ is the present value of the Hubble function. Then from Eqs. (35) and (38), we get

$\frac{H^{2}}{H_{0}^{2}}=\Omega_{\mathrm{m}}+\Omega_{\mathrm{de}}$

and

$\dot{\Omega}_{\mathrm{m}}=-3 H \Omega_{\mathrm{m}}-\dot{\Omega}_{\mathrm{de}}$.

The above equations are sufficient to find the behavior of the matter, the dark energy, the Hubble function and the scale factor as a function of cosmological time. We cannot find the exact solutions because these equations are too complicated. In this case we should search for numerical solutions. The behavior of the dark energy is presented in Figs. 2 and 3 . Figure 2 shows the diagram of the dependence $\Omega_{\mathrm{de}}(\tau)$ with respect of the rescaled time $\tau$ for $\alpha=10^{-105}$ and $\frac{E_{0}}{3 H_{0}^{2}}=10^{120}$. On the diagram we can see that the start value of the dark energy density, which is equal to $\Omega_{\mathrm{de}} \approx 10^{120}$, is reduced to the present value of the dark energy density, which is $\Omega_{\mathrm{de}} \approx 0.7$. This final value of $\Omega_{\mathrm{de}}$ does not depend on the values of the parameters $\alpha$ and $\frac{E_{0}}{3 H_{0}^{2}}$. Therefore, this mechanism makes an attempt of solving the cosmological

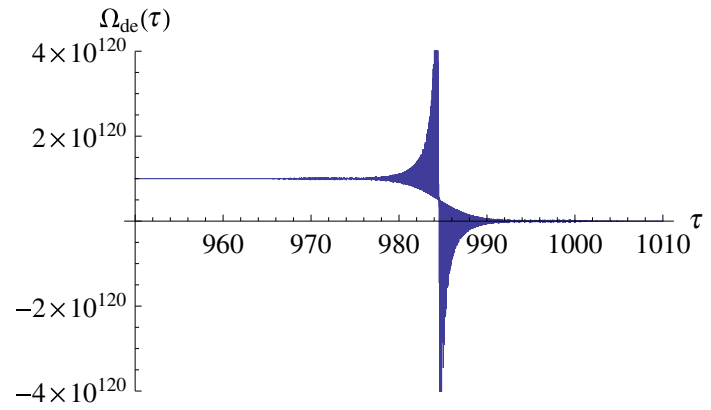

Fig. 2 The dependence $\Omega_{\mathrm{de}}(\tau)$ for $\alpha=10^{-105}$ and $\frac{E_{0}}{3 H_{0}^{2}}=10^{120}$. The rescaled time $\tau$ is given in units of $\left[1.3 \times 10^{-40} s\right]$ 


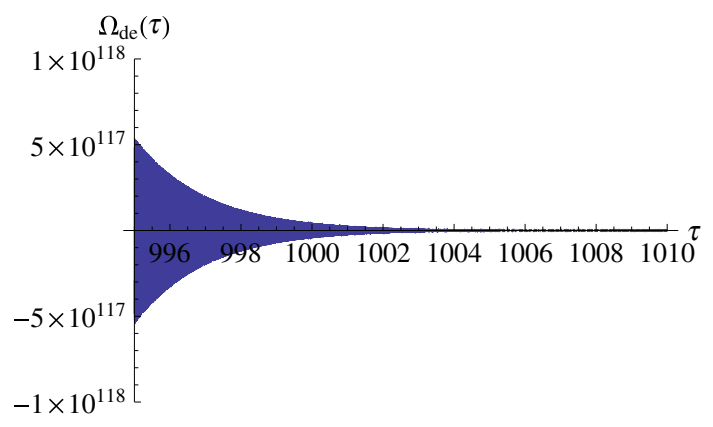

Fig. 3 The dependence $\Omega_{\mathrm{de}}(\tau)$ during the intermediate phase of damped oscillations for $\alpha=10^{-105}$ and $\frac{E_{0}}{3 H_{0}^{2}}=10^{120}$. The rescaled time $\tau$ is given in units of $\left[1.3 \times 10^{-40} s\right]$

constant problem. For late time, dark energy can be treated as the cosmological constant. The characteristic of the intermediate oscillatory regime is depending on the parameter $\alpha$. With the increasing value of $\alpha$ the number of oscillations, their amplitude, their period as well as the length of this regime decreases. If $\alpha>0.4$ then oscillations begin to disappear and the value of $\Omega_{\text {de }}$ jumps to the constant value of 0.7 .

Figure 3 shows the diagram of the dependence $\Omega_{\mathrm{de}}(\tau)$ during the intermediate phase of damped oscillations with respect of the time $\tau$ for $\alpha=10^{-105}$ and $\frac{E_{0}}{3 H_{0}^{2}}=10^{120}$. Note that the dark energy oscillates and the amplitude of the oscillations decreases with time. In consequence the dark energy can be treated as the cosmological constant after the intermediate phase of oscillations. Figure 4 shows the diagrams of the dependence $\Omega_{\mathrm{de}}(\tau)$ with respect of the time $\tau$ for different values of $\alpha(\alpha=0.2,0.4,0.8)$ and $\frac{E_{0}}{3 H_{0}^{2}}=10^{120}$. This figure presents how the evolution of $\Omega_{\mathrm{de}}(\tau)$ is dependent on the parameter $\alpha$. Note that the oscillations disappear for $\alpha>0.4$.

In general, if $\alpha$ decreases then the times when oscillatory regime takes place increase. This means that passage from the very high energies to the extremely small energies, which takes place at the oscillatory regime, moves in the direction of increasing time with decreasing $\alpha$ and for a suitable small value of $\alpha$ this oscillatory regime can occur at relatively late times.

Figure 5 presents the evolution of $\frac{d \Omega_{\mathrm{de}}}{d \tau}$. The evolution of matter is demonstrated in Fig. 6 and the Hubble function is presented in Fig. 7. The diagram of the scale factor with respect to the cosmological time is presented in Fig. 8 .

We have $\tau=\frac{\alpha\left(E_{0}-\Lambda_{\text {bare }}\right)}{\hbar(1-\alpha)} V_{0} t$; therefore if the value of the parameter $\alpha$ increases then the damping of oscillations should also be increased. In the limiting case, if $\alpha$ is equal zero then we get the $\Lambda \mathrm{CDM}$ model. This last conclusion can easily be drawn analyzing the late time properties of $\rho_{\text {de. }}$.

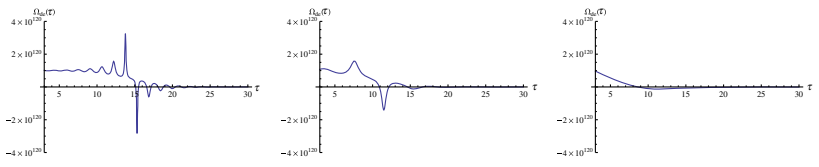

Fig. 4 The dependence $\Omega_{\mathrm{de}}(\tau)$ for $\alpha=0.2$ (left figure) and $\alpha=0.4$ (medium figure) and $\alpha=0.8$ (right figure) and $\frac{E_{0}}{3 H_{0}^{2}}=10^{120}$. The rescaled time $\tau$ for the left figure is given in units of $\left[5.3 \times 10^{-145} s\right]$, for the center figure is given in units of $\left[2.0 \times 10^{-145} \mathrm{~s}\right]$ and for the right figure is given in units of $\left[3.3 \times 10^{-146} s\right]$

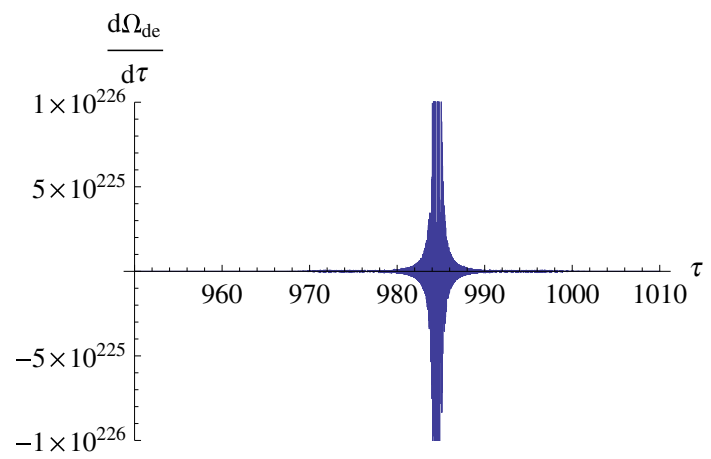

Fig. 5 The dependence $\frac{\mathrm{d} \Omega_{\mathrm{de}}}{\mathrm{d} \tau}(\tau)$ for $\alpha=10^{-105}$ and $\frac{E_{0}}{3 H_{0}^{2}}=10^{120}$. Note that, for negative value of $\frac{\mathrm{d} \Omega_{\mathrm{de}}}{\mathrm{d} \tau}$, the energy is transferred from the dark energy to the dark matter and for the positive value of $\frac{d \Omega_{\mathrm{de}}}{\mathrm{d} \tau}$, the energy is transfered from the dark matter to the dark energy. The rescaled time $\tau$ is given in unit $\left[1.3 \times 10^{-40} s\right]$

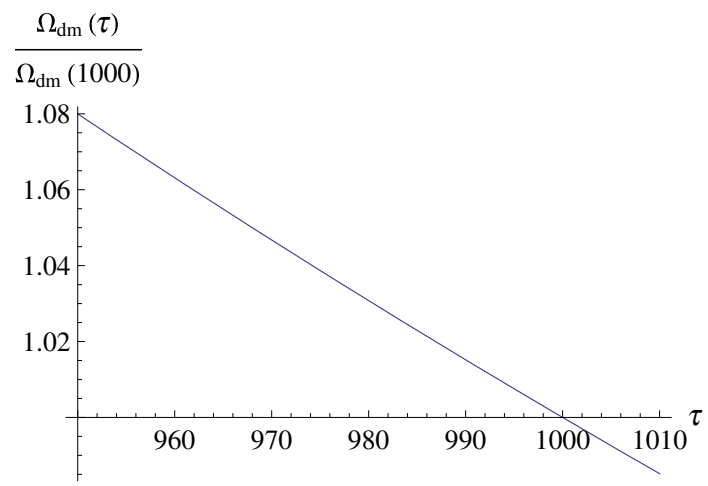

Fig. 6 The dependence $\Omega_{\mathrm{dm}}$ for $\alpha=10^{-105}$ and $\frac{E_{0}}{3 H_{0}^{2}}=10^{120}$. We include the influence of the radiation for the evolution of the matter. Note that the dark energy has a negligible influence on the evolution of the matter. The rescaled time $\tau$ is given in units of $\left[1.3 \times 10^{-40} s\right]$

For the late time, $\tau \rightarrow \infty$, according to Eq. (28), the parametrization of dark energy (31) can be approximated by the following expression:

$\rho_{\mathrm{de}}=\Lambda_{\text {bare }}-2 E_{\mathrm{R}} \frac{\alpha^{2}}{(1-\alpha)^{2}+\frac{\alpha^{2}}{4}} \frac{1}{\tau^{2}}+\cdots$

From this relation the important observation follows: For any $\alpha>0$ the $\Lambda \mathrm{CDM}$ model is the limiting case, when 


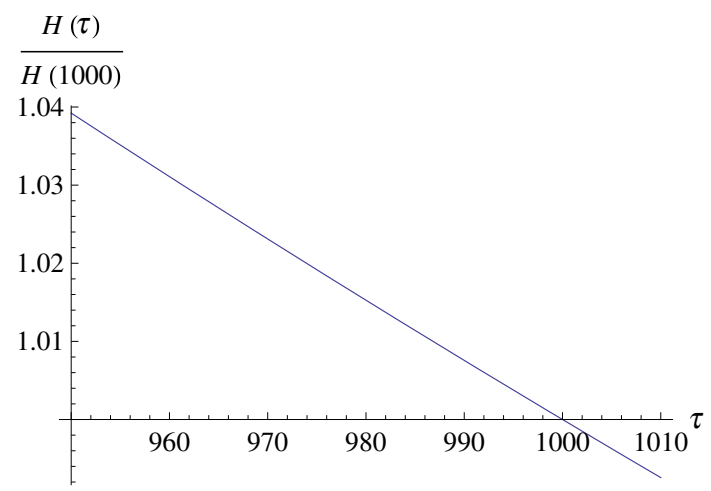

Fig. 7 The dependence $H(\tau)$ for $\alpha=10^{-105}$ and $\frac{E_{0}}{3 H_{0}^{2}}=10^{120}$. We include influence of the radiation for the evolution of the Hubble function. Note that dark energy has a negligible influence on the evolution of the Hubble function. The rescaled time $\tau$ is given in units of $\left[1.3 \times 10^{-40} s\right]$

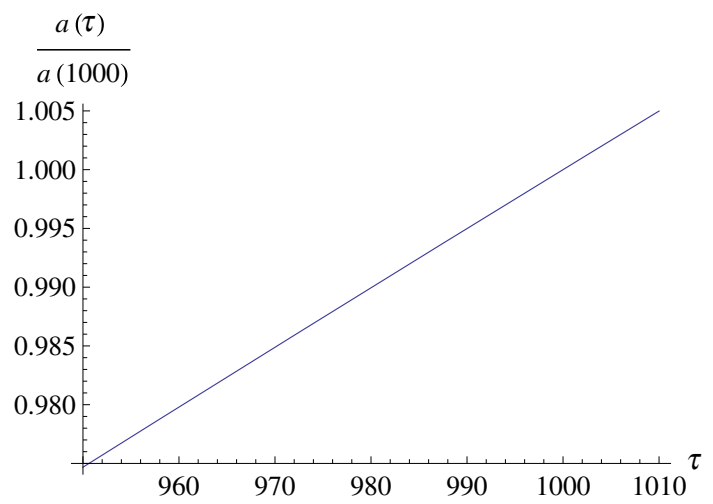

Fig. 8 The dependence $a(\tau)$ for $\alpha=10^{-105}$ and $\frac{E_{0}}{3 H_{0}^{2}}=10^{120}$. We include the influence of the radiation on the evolution of the scale factor. Note that dark energy has negligible influence on the evolution of the scale factor. The rescaled time $\tau$ is given in units of $\left[1.3 \times 10^{-40} s\right]$

$\tau \rightarrow \infty$, of our model. So for very, very late times the results obtained within our model and within the $\Lambda$ CDM model have to coincide. This parametrization of the dark energy was considered in $[52,54,55]$.

The dark energy is significantly lower than the energy density of matter in the early Universe, which has the consequence that the transfer to the dark sector is negligible (see Fig. 5). Our model makes an attempt of solving the cosmological constant problem. In general, the amplitude of oscillations of the dark energy decreases with time.

Thus for the late time Universe, oscillations are negligible and the dark energy has the form of the cosmological constant.

The conservation equation for the dark energy (41) can be rewritten as

$\dot{\rho}_{\mathrm{de}}=-3 H\left(\rho_{\mathrm{de}}+p_{\mathrm{de}}\right)$,

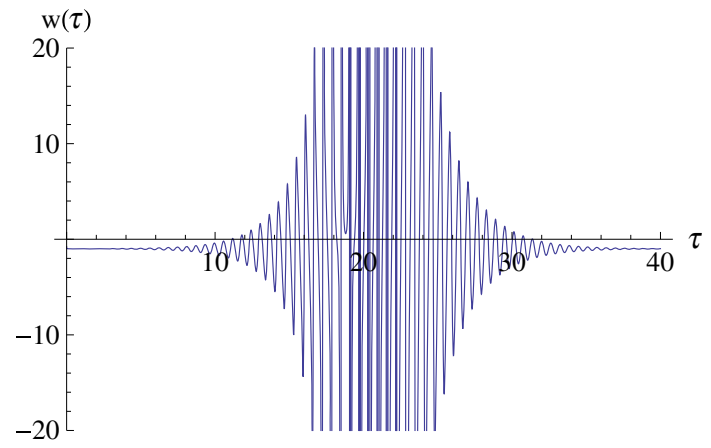

Fig. 9 The typical dependence $w(\tau)$. This example is for $\alpha=0.09$ and $\frac{E_{0}}{3 H_{0}^{2}}=10^{120}$. Note that after the intermediate phase of oscillations, the function $w(\tau)$ can be treated as a constant, which is equal to -1 . The rescaled time $\tau$ is given in units of $\left[1.3 \times 10^{-144} s\right]$

where $p_{\text {de }}$ is an effective pressure of the dark energy. In this case the equation of state for the dark energy is expressed by the following formula:

$p_{\mathrm{de}}=w(t) \rho_{\mathrm{de}}$,

where the function $w(t)$ is given by the expression

$w(t)=-1-\frac{\dot{\rho}_{\mathrm{de}}}{\sqrt{3} \sqrt{\rho_{\mathrm{m}}+\rho_{\mathrm{de}}} \rho_{\mathrm{de}}}=-1-\frac{1}{3 H} \frac{\mathrm{d} \ln \rho_{\mathrm{de}}}{\mathrm{d} t}$.

The diagram of coefficient equation of state $w(t)$ is presented in Fig. 9. The function $w(t)$, for the late time, is a constant and equals -1 , which means that it describes the cosmological constant parameter. Note that the function $w(t)$ is also equal -1 , which means that $\rho_{d e}$ is constant as a consequence of the conservation condition (transfer between the sectors is negligible). Therefore, the energy transfer is an effective process only during the intermediate oscillation period (quantum regime).

Let $\rho_{\mathrm{de}} \gg \rho_{\mathrm{m}}$. Then our model predicts inflation. The formula for the e-foldings $N=H_{\text {init }}\left(t_{\text {fin }}-t_{\text {init }}\right)$ (see [56]) becomes the following expression for our model:

$N=\sqrt{\frac{E_{0}}{3}}\left(t_{\text {fin }}-t_{\text {init }}\right)$,

where $t_{\text {init }} \approx 0$ and $t_{\text {fin }}$ is the time of appearing of the intermediate phase of oscillations. Figure 10 presents the evolution of the scale factor $a$ with respect to the cosmological time during inflation.

\section{Statistical analysis}

To estimate the model parameters we use the astronomical observations such as the supernovae of type Ia (SNIa), BAO, 


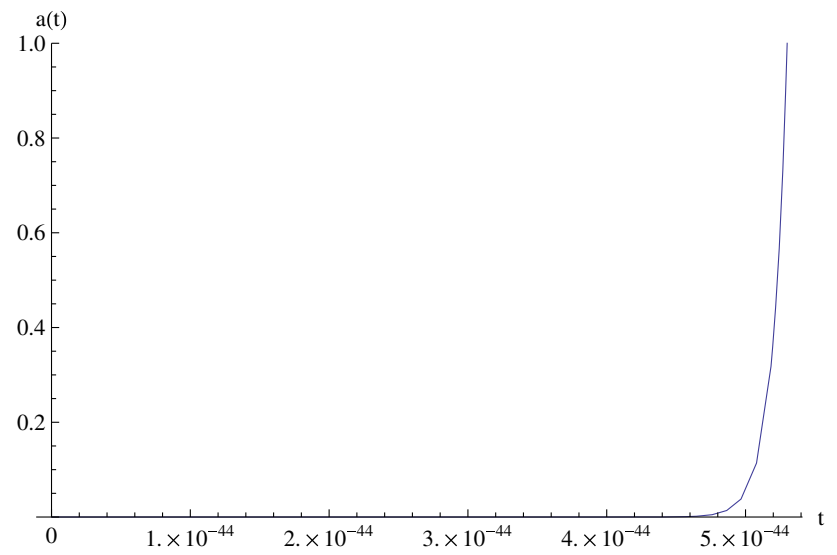

Fig. 10 The dependence $a(t)$ for $\frac{E_{0}}{3 H_{0}^{2}}=2 * 10^{125}$. We assume that $\rho_{\mathrm{de}} \gg \rho_{\mathrm{m}}$ and the intermediate phase of oscillations is after the Planck epoch. Note that, for the above assumptions, inflation appears after the Planck epoch. The characteristic number of e-foldings of this inflation is equal to 53 here. The cosmological time $t$ is given in seconds

measurements of $H(z)$ for galaxies, the Alcock-Paczyński test and the measurements CMB.

The data of supernovae of type Ia, which were used in this paper, are taken from the Union 2.1 dataset [57]. In this context we use the following likelihood function:

$\ln L_{\mathrm{SNIa}}=-\frac{1}{2}\left[A-B^{2} / C+\log (C /(2 \pi))\right]$,

where $A=\left(\mu^{\text {obs }}-\mu^{\text {th }}\right) \mathbb{C}^{-1}\left(\mu^{\text {obs }}-\mu^{\text {th }}\right), B=\mathbb{C}^{-1}\left(\mu^{\text {obs }}-\right.$ $\left.\mu^{\text {th }}\right), C=\operatorname{Tr} \mathbb{C}^{-1}$ and $\mathbb{C}$ is a covariance matrix for SNIa. The observer distance modulus $\mu^{\text {obs }}$ is defined by the formula $\mu^{\text {obs }}=m-M$ (where $m$ is the apparent magnitude and $M$ is the absolute magnitude of SNIa). The theoretical distance modulus is given by $\mu^{\text {th }}=5 \log _{10} D_{L}+25$ (where the luminosity distance is $\left.D_{L}=c(1+z) \int_{0}^{z} \frac{d z^{\prime}}{H(z)}\right)$.

We use the following BAO data: Sloan Digital Sky Survey Release 7 (SDSS DR7) dataset at $z=0.275$ [58], 6dF Galaxy Redshift Survey measurements at redshift $z=0.1$ [59], and WiggleZ measurements at redshift $z=0.44,0.60,0.73$ [60]. The likelihood function is defined by the expression

$\ln L_{\mathrm{BAO}}=-\frac{1}{2}\left(\mathbf{d}^{\mathrm{obs}}-\frac{r_{s}\left(z_{d}\right)}{D_{V}(\mathbf{z})}\right) \mathbb{C}^{-1}\left(\mathbf{d}^{\mathrm{obs}}-\frac{r_{s}\left(z_{d}\right)}{D_{V}(\mathbf{z})}\right)$,

where $r_{s}\left(z_{d}\right)$ is the sound horizon at the drag epoch [61,62].

Measurements of the Hubble parameter $H(z)$ of galaxies were taken from [63-65]. The likelihood function is given by the following formula:

$\ln L_{H(z)}=-\frac{1}{2} \sum_{i=1}^{N}\left(\frac{H\left(z_{i}\right)^{\mathrm{obs}}-H\left(z_{i}\right)^{\mathrm{th}}}{\sigma_{i}}\right)^{2}$.
The likelihood function for the Alcock-Paczynski test [66, 67] has the following form:

$\ln L_{A P}=-\frac{1}{2} \sum_{i} \frac{\left(A P^{t h}\left(z_{i}\right)-A P^{o b s}\left(z_{i}\right)\right)^{2}}{\sigma^{2}}$,

where $A P(z)^{\text {th }} \equiv \frac{H(z)}{z} \int_{0}^{z} \frac{\mathrm{d} z^{\prime}}{H\left(z^{\prime}\right)}$ and $A P\left(z_{i}\right)^{\text {obs }}$ are observational data [68-76].

In this paper, the likelihood function for the measurements of CMB [77] and lensing by Planck, and low- $\ell$ polarization from the WMAP (WP), has the following form:

$\ln L_{\mathrm{CMB}+\text { lensing }}=-\frac{1}{2}\left(\mathbf{x}^{\mathrm{th}}-\mathbf{x}^{\mathrm{obs}}\right) \mathbb{C}^{-1}\left(\mathbf{x}^{\mathrm{th}}-\mathbf{x}^{\mathrm{obs}}\right)$,

where $\mathbb{C}$ is the covariance matrix with the errors, $\mathbf{x}$ is a vector of the acoustic scale $l_{A}$, the shift parameter $R$ and $\Omega_{b} h^{2}$ where

$l_{A}=\frac{\pi}{r_{S}\left(z^{*}\right)} c \int_{0}^{z^{*}} \frac{\mathrm{d} z^{\prime}}{H\left(z^{\prime}\right)}$

$R=\sqrt{\Omega_{\mathrm{m}, 0} H_{0}^{2}} \int_{0}^{z^{*}} \frac{\mathrm{d} z^{\prime}}{H\left(z^{\prime}\right)}$,

where $z^{*}$ is the redshift of the epoch of the recombination [61].

In this paper, the final formula for the likelihood function is given in the following form:

$L_{\mathrm{tot}}=L_{\mathrm{SNIa}} L_{\mathrm{BAO}} L_{\mathrm{AP}} L_{H(z)} L_{\mathrm{CMB}+\text { lensing }}$.

The statistical analysis was done by our own code CosmoDarkBox. This code uses the Metropolis-Hastings algorithm $[78,79]$.

We estimated four cosmological parameters: $H_{0}, \Omega_{\mathrm{m}, 0}, \alpha$ and the parameter $E_{0}$. Our statistical results are completed in Table 1. We present intersections of the likelihood function with 68 and 95\% confidence level projections in Figs. 11, 12,13 and 14. PDF diagrams for $\alpha$ and $\frac{E_{0}}{3 H_{0}^{2}}$ are presented in Figs. 15 and 16.

The values of the likelihood function are not always sensitive to changing of the parameters $\alpha$ and $E_{0}$. The possible changing of the values of the likelihood function are beyond

Table 1 The best fit and errors for the estimated model for SNIa + BAO $+H(z)+\mathrm{AP}+\mathrm{CMB}$ test with $H_{0}$ from the interval $(66.0,72.0), \Omega_{\mathrm{m}, 0}$ from the interval $(0.27,0.34) . \Omega_{\mathrm{b}, 0}$ is assumed as 0.048468

\begin{tabular}{llll}
\hline Parameter & Best fit & $68 \% \mathrm{CL}$ & $95 \% \mathrm{CL}$ \\
\hline$H_{0}$ & $68.82 \mathrm{~km} /(\mathrm{s} \mathrm{Mpc})$ & +0.61 & +0.98 \\
& & -0.55 & -0.92 \\
$\Omega_{\mathrm{m}, 0}$ & 0.3009 & +0.0079 & +0.0133 \\
& & -0.0084 & -0.0134 \\
\hline
\end{tabular}




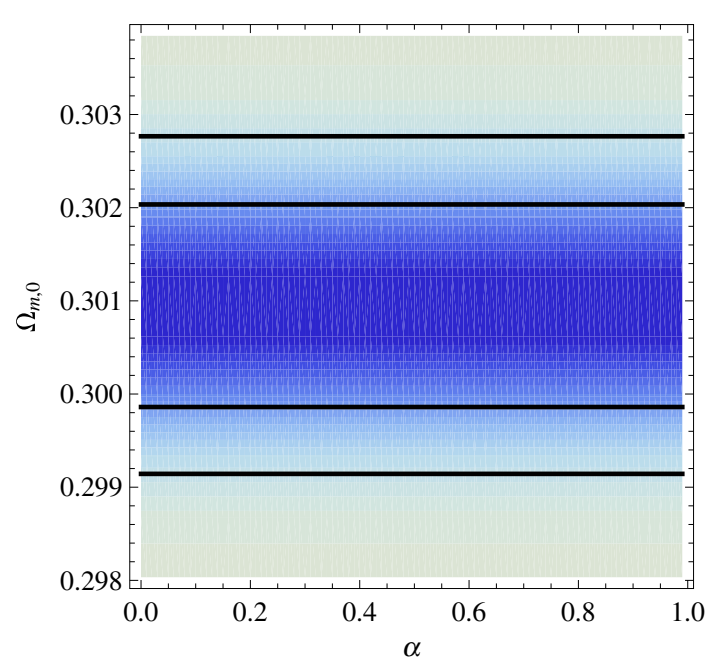

Fig. 11 The intersection of the likelihood function of two model parameters $\left(\Omega_{\mathrm{m}, 0}, \alpha\right)$, with the marked 68 and $95 \%$ confidence levels. The plane of the intersection is the best fit of $H_{0}\left(H_{0}=68.82\left[\frac{\mathrm{km}}{\mathrm{s} \times \mathrm{Mpc}}\right]\right)$. We assumed that $E_{0} /\left(3 H_{0}^{2}\right)$ is equal to $10^{120}$, but changing of the value of $E_{0} /\left(3 H_{0}^{2}\right)$ does not influence the results. Note that the values of the likelihood function are not sensitive to changing of the parameter $\alpha$

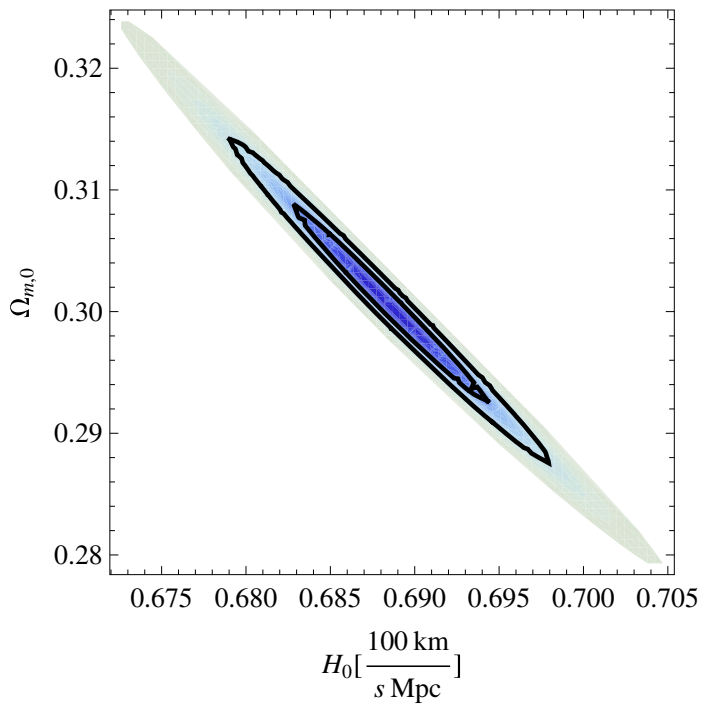

Fig. 12 The intersection of the likelihood function of two model parameters $\left(\Omega_{\mathrm{m}, 0}, H_{0}\right)$, with the marked 68 and $95 \%$ confidence levels. The plane of the intersection is $\alpha=0.5$ and $E_{0}=10^{120}$

the capabilities of numerical methods. This fact can be interpreted as the lack of sensitivity of the present evolution of the Universe for changing of the parameters $\alpha$ and $E_{0}$. The best fit values of $H_{0}$ and $\Omega_{\mathrm{m}}$ for our model are equivalent of the best fit values for the $\Lambda \mathrm{CDM}$ model.

\section{Conclusion}

The main goal of our paper was to analyze the cosmological model with the running dark energy as well as the dark matter

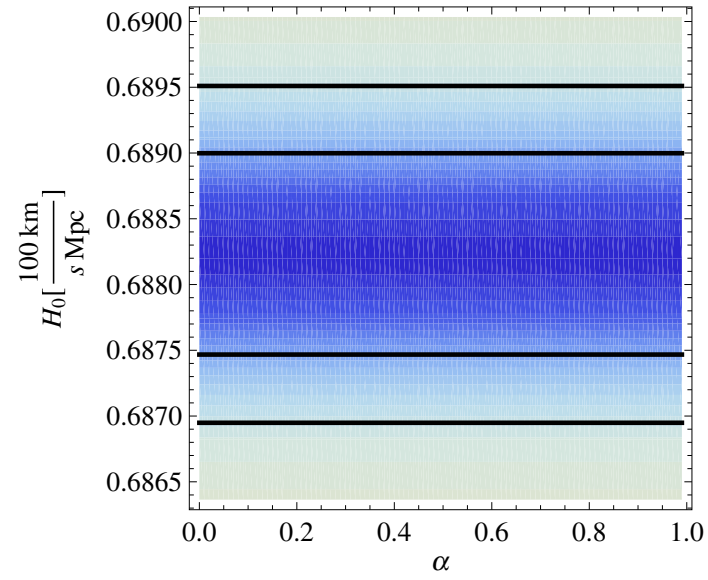

Fig. 13 The intersection of the likelihood function of two model parameters $\left(H_{0}, \alpha\right)$, with the marked 68 and $95 \%$ confidence levels. The plane of the intersection is the best fit of $\Omega_{\mathrm{m}, 0}\left(\Omega_{\mathrm{m}, 0}=0.3009\right)$. We assumed that $E_{0} /\left(3 H_{0}^{2}\right)$ is equal to $10^{120}$, but changing of the value of $E_{0} /\left(3 H_{0}^{2}\right)$ does not influence the results. Note that the values of the likelihood function are not sensitive to changing of the parameter $\alpha$

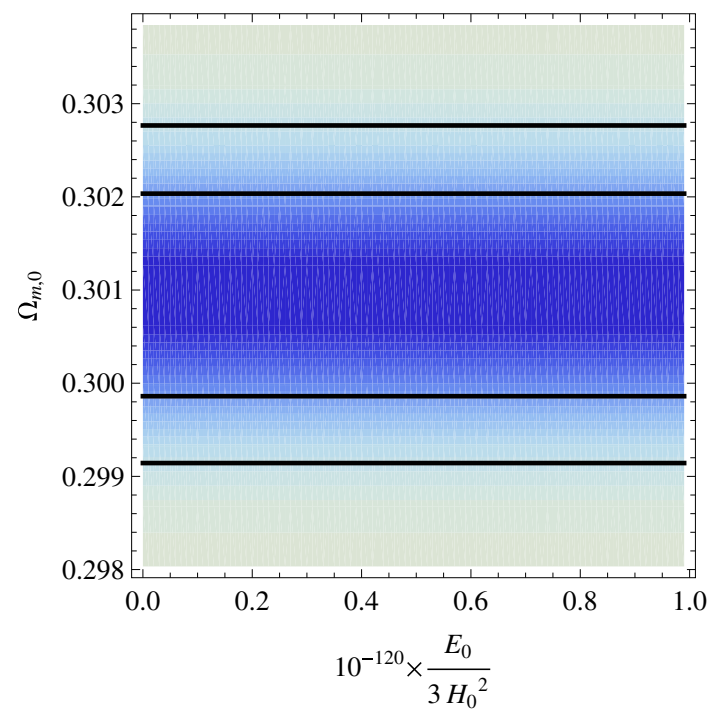

Fig. 14 The intersection of the likelihood function of two model parameters $\left(\Omega_{\mathrm{m}, 0}, \frac{E_{0}}{3 H_{0}^{2}}\right)$, with the marked 68 and $95 \%$ confidence levels. The plane of the intersection is the best fit of $H_{0}\left(H_{0}=68.82\left[\frac{\mathrm{km}}{\mathrm{s} \times \mathrm{Mpc}}\right]\right)$. We assumed that $\alpha$ is equal to 0.1 , but changing of the value of $\alpha$ does not influence the results. Note that the values of the likelihood function are not sensitive to changing of $\frac{E_{0}}{3 H_{0}^{2}}$

and the baryonic matter in the form of dust. We considered the evolution of the dark energy using the fact that the decay of a false vacuum to the true vacuum is a quantum decay process. From the cosmological point of view this model was formulated in terms of the cosmological model with the interaction between dark matter and dark energy. 


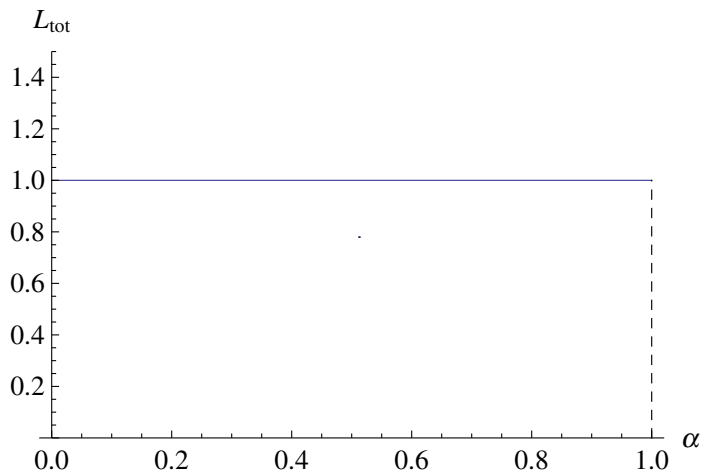

Fig. 15 Diagram of PDF for parameter $\alpha$ obtained as an intersection of the likelihood function. Two planes of the intersection likelihood function are $H_{0}=68.82\left[\frac{\mathrm{km}}{\mathrm{s} \times \mathrm{Mpc}}\right]$ and $\Omega_{\mathrm{m}, 0}=0.3009$. The planes of intersection are constructed from the best fitting value of the model parameters. We assume the value of $\alpha$ from the interval $(0,1)$. Note that the values of the likelihood function are not sensitive to changing of $\alpha$

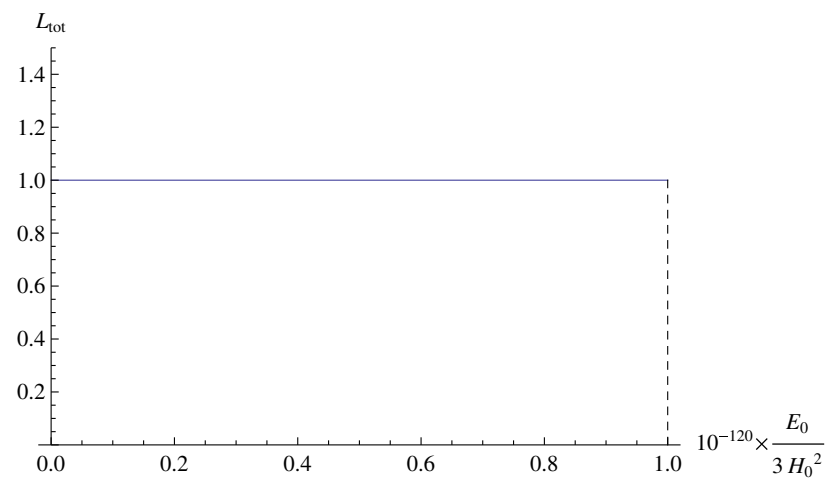

Fig. 16 Diagram of PDF for $\frac{E_{0}}{3 H_{0}^{2}}$ obtained as an intersection of the likelihood function. Two planes of intersection likelihood function are $H_{0}=68.82\left[\frac{\mathrm{km}}{\mathrm{s} \times \mathrm{Mpc}}\right]$ and $\Omega_{\mathrm{m}, 0}=0.3009$. The planes of intersection are constructed from the best fitting value of the model parameters. We assume the value of $\frac{E_{0}}{3 H_{0}^{2}}$ from the interval $\left(0,10^{120}\right)$. Note that the values of the likelihood function are not sensitive to changing of $\frac{E_{0}}{3 H_{0}^{2}}$

We detected the intermediate phase of oscillations between phases of constant dark energy. The preceding phase has $\rho_{\mathrm{de}}=E_{0}$ and the following phase has $\rho_{\mathrm{de}}=\Lambda_{\text {bare }}$. Defining this class of models parametrized with $\alpha$ (the deviation from the $\Lambda$ CDM model) we have found two different types of dynamical behavior. Independently of $0<\alpha<1$ there is a universal mechanism of jumping of the value of energy density of dark energy from the initial value of $E_{0}=10^{120}$ to the present value of the cosmological constant of 0.7 .

During this epoch there is the oscillatory behavior of energy density of dark energy as well as its coefficient equation of state. In this intermediate regime the amplitude of the oscillations increases, then there is a jump down followed by the decreasing oscillations. This kind of oscillation appears for $0<\alpha<0.4$. The number, period and amplitude of oscillations as well as the length of this intermediate regime decreases as the parameter $\alpha$ grows. For $\alpha>0.4$ the oscillations disappear and only the jump down of energy density of dark energy remains. The jump down mechanism is independent from the value of the parameter $\alpha$, which leads to solving the cosmological constant problem.

In the early Universe the energy density of dark energy is significantly lower than the energy density of dark matter, therefore the change of energy density of the dark matter, which is caused by energy transfer in the dark sector, is negligible.

While our model makes an attempt of an explanation of the cosmological constant problem, the coincidence problem is still open as we forced the model to have an exit on the present value of the cosmological constant. In the early Universe, the dark energy oscillates. But the amplitude of the oscillations decreases with time. In consequence, for the late time Universe, oscillations are negligible and the dark energy can be described as the cosmological constant. Unfortunately our model cannot explain why the present value of dark energy and matter are comparable.

From the statistical analysis of the model we found that the model is generic in the sense that independently from the values of the parameters $\alpha$ and $E_{0}$ we can obtain the present value of the energy density of the dark energy. Therefore, the $\triangle \mathrm{CDM}$ model is an attractor which all models with different values of parameters $\alpha$ and $E_{0}$ can reach. The final interval of evolution for which we have data at our disposal is identical for a whole class of models, therefore it is impossible to find best-fitted values of the model parameters and indicate one particular model (degeneration problem).

As should be expected it is difficult to discriminate the parameters of the early state of the Universe as there is no data for very high redshift. In Figs. 15 and 16 the likelihood functions for parameters of interest are flat, so there is no best fit value. That is why we take calibrated values of these parameters for further analysis in this paper. We assume that the false vacuum energy is $10^{120}$ as is indicated from the quantum field theory. On the other hand the parameter $\alpha$ should be chosen to get the decaying process of false vacuum to take place after the Planck era.

Acknowledgements The work has been supported by Polish National Science Centre (NCN), Project DEC-2013/09/B/ ST2/03455.

Open Access This article is distributed under the terms of the Creative Commons Attribution 4.0 International License (http://creativecomm ons.org/licenses/by/4.0/), which permits unrestricted use, distribution, and reproduction in any medium, provided you give appropriate credit to the original author(s) and the source, provide a link to the Creative Commons license, and indicate if changes were made.

Funded by $\mathrm{SCOAP}^{3}$. 


\section{References}

1. J. Polchinski, in The Quantum Structure of Space and Time: Proceedings of the 23rd Solvay Conference on Physics. Brussels, Belgium. 1-3 Dec 2005 (2006), pp. 216-236

2. S. Weinberg, Rev. Mod. Phys. 61, 1 (1989). doi:10.1103/ RevModPhys.61.1

3. S.M. Carroll, W.H. Press, E.L. Turner, Ann. Rev. Astron. Astrophys. 30, 499 (1992). doi:10.1146/annurev.aa.30.090192.002435

4. A.D. Dolgov, in Phase transitions in cosmology. Proceedings, 4th Cosmology Colloquium, Euroconference, Paris, France (1997), pp. 161-175. http://alice.cern.ch/format/showfull?sysnb=0254991. Accessed date 4-9 June 1997

5. V. Sahni, A.A. Starobinsky, Int. J. Mod. Phys. D 9, 373 (2000)

6. N. Straumann, Eur. J. Phys. 20, 419 (1999). doi:10.1088/ 0143-0807/20/6/307

7. S. Weinberg, in Sources and detection of dark matter and dark energy in the universe. Proceedings, 4th International Symposium, DM 2000, Marina del Rey, USA (2000), pp. 18-26. http://www.slac.stanford.edu/spires/find/books/www? $\mathrm{cl}=\mathrm{QB} 461: \mathrm{I57:2000}$. Accessed date 23-25 Feb 2000

8. S.M. Carroll, Living Rev. Rel. 4, 1 (2001). doi:10.12942/lrr-2001-1

9. S.E. Rugh, H. Zinkernagel, Stud. Hist. Philos. Sci. B33, 663 (2002). doi:10.1016/S1355-2198(02)00033-3

10. T. Padmanabhan, Phys. Rept. 380, 235 (2003). doi:10.1016/ S0370-1573(03)00120-0

11. J. Yokoyama, in Proceedings, 12th Workshop on General Relativity and Gravitation (JGRG12): Tokyo, Japan (2003). http:// alice.cern.ch/format/showfull?sysnb=2376595. Accessed date 2528 Nov 2002

12. S. Sarkar, Curr. Sci. 88, 2120 (2005)

13. E.J. Copeland, M. Sami, S. Tsujikawa, Int. J. Mod. Phys. D 15, 1753 (2006). doi:10.1142/S021827180600942X

14. M. Szydlowski, Phys. Rev. D 91(12), 123538 (2015). doi:10.1103/ PhysRevD.91.123538

15. R. Bousso, D. Harlow, L. Senatore, Phys. Rev. D 91(8), 083527 (2015). doi:10.1103/PhysRevD.91.083527

16. R. Bousso, D. Harlow, L. Senatore, JCAP 1412(12), 019 (2014). doi:10.1088/1475-7516/2014/12/019

17. S.R. Coleman, Phys. Rev. D 15, 2929 (1977). doi:10.1103/ PhysRevD.15.2929. doi:10.1103/PhysRevD.16.1248. [Erratum: Phys. Rev. D 16, 1248 (1977)]

18. C.G. Callan Jr., S.R. Coleman, Phys. Rev. D 16, 1762 (1977). doi:10.1103/PhysRevD.16.1762

19. S.R. Coleman, F. De Luccia, Phys. Rev. D 21, 3305 (1980). doi:10. 1103/PhysRevD.21.3305

20. A. Kobakhidze, A. Spencer-Smith, Phys. Lett. B 722, 130 (2013). doi:10.1016/j.physletb.2013.04.013

21. G. Degrassi, S. Di Vita, J. Elias-Miro, J.R. Espinosa, G.F. Giudice, G. Isidori, A. Strumia, JHEP 08, 098 (2012). doi:10.1007/ JHEP08(2012)098

22. J. Elias-Miro, J.R. Espinosa, G.F. Giudice, G. Isidori, A. Riotto, A. Strumia, Phys. Lett. B 709, 222 (2012). doi:10.1016/j.physletb. 2012.02.013

23. W. Chao, M. Gonderinger, M.J. Ramsey-Musolf, Phys. Rev. D 86, 113017 (2012). doi:10.1103/PhysRevD.86.113017

24. L.M. Krauss, J. Dent, Phys. Rev. Lett. 100, 171301 (2008). doi:10. 1103/PhysRevLett.100.171301

25. L.M. Krauss, J. Dent, G.D. Starkman, Int. J. Mod. Phys. D 17, 2501 (2009). doi:10.1142/S021827180801400X

26. S. Winitzki, Phys. Rev. D 77, 063508 (2008). doi:10.1103/ PhysRevD.77.063508

27. R.G. Landim, E. Abdalla, Phys. Lett. B 764, 271 (2017). doi:10. 1016/j.physletb.2016.11.044
28. NIST Handbook of Mathematical Functions, ed. by F. W. J. Olver, D. W. Lozier, R. F. Boisvert, C. W. Clark, National Institute of Standards and Technology, Cambridge University Press, Cambridge (2010)

29. Handbook of Mathematical Functions, ed. by M. Abramowitz, I. A. Stegun, National Bureau of Standards, Appl. Math. Ser. No. 55, U.S. G.P.O., Washington, D.C. (1964)

30. N.S. Krylov, V.A. Fock, Z. Eksp, Teor. Fiz. 17, 93 (1947). [in Russian]

31. V.A. Fock, Fundamentals of Quantum Mechanics (Mir Publishers, Moscow, 1978)

32. L. Fonda, G.C. Ghirardi, A. Rimini, Rept. Progr. Phys. 41, 587 (1978). doi:10.1088/0034-4885/41/4/003

33. L.A. Khalfin, Z. Eksp, Teor. Fiz. (USSR) 33, 1371 (1957). [in Russian]

34. L.A. Khalfin, Z. Eksp, Sov. Phys. JETP 6, 1053 (1958)

35. N.G. Kelkar, M. Nowakowski, J. Phys. A 43, 385308 (2010). doi:10.1088/1751-8113/43/38/385308

36. J. Martorell, J. G. Muga, D. W. L. Sprung, in Time in Quantum Mechanics Vol. 2, ed. by G. Muga, A. Ruschhaupt, and A. del Campo, Lect. Notes Phys., 789, Springer, Berlin, p. 329 (2009)

37. E. Torrontegui, J.G. Muga, J. Martorell, D.W.L. Sprung, Adv. Quant. Chem. 60, 485 (2010)

38. G. Garcia-Calderon, R. Romo, J. Villavicencio, Phys. Rev. B 76, 035340 (2007)

39. F. Giraldi, Eur. Phys. J. D 69, 5 (2015)

40. F. Giacosa, Found. Phys. 42, 1262 (2012). doi:10.1007/ s10701-012-9667-3

41. M.L. Goldberger, K.M. Watson, Collision Theory (Wiley, New York, NY, 1964)

42. K. Urbanowski, Phys. Rev. A 50, 2847 (1994)

43. K. Urbanowski, Eur. Phys. J. C 58, 151 (2008). doi:10.1140/epjc/ s10052-008-0725-0

44. K. Urbanowski, Cent. Eur. J. Phys. 7, 696 (2009). doi:10.2478/ s11534-009-0053-5

45. K. Urbanowski, Theor. Math. Phys. 190(3), 458 (2017). doi:10. 1134/S0040577917030151

46. K. Urbanowski, K. Raczynska, Phys. Lett. B 731, 236 (2014). doi:10.1016/j.physletb.2014.02.043

47. V. Branchina, E. Messina, Phys. Rev. Lett. 111, 241801 (2013). doi:10.1103/PhysRevLett.111.241801

48. F. Bezrukov, M. Shaposhnikov, C. R. Phys. 16, 994 (2015). doi:10. 1016/j.crhy.2015.08.005

49. K. Urbanowski, Phys. Rev. Lett. 107, 209001 (2011). doi:10.1103/ PhysRevLett.107.209001

50. K. Urbanowski, Eur. Phys. J. D 54, 25 (2009)

51. K. Urbanowski, M. Szydlowski, AIP Conf. Proc. 1514, 143 (2012). doi:10.1063/1.4791743

52. M. Szydlowski, A. Stachowski, K. Urbanowski, J. Phys. Conf. Ser. 626, 012033 (2015)

53. O. Minazzoli, T. Harko, Phys. Rev. D 86, 087502 (2012). doi:10. 1103/PhysRevD.86.087502

54. M. Szydlowski, A. Stachowski, JCAP 1510(10), 066 (2015) doi:10.1088/1475-7516/2015/10/066

55. M. Szydlowski, A. Stachowski, Phys. Dark Univ. 15, 96 (2017). doi:10.1016/j.dark.2017.01.002

56. A. De Felice, S. Tsujikawa, Living Rev. Rel. 13, 3 (2010). doi:10. 12942/lrr-2010-3

57. N. Suzuki et al., Astrophys. J. 746, 85 (2012). doi:10.1088/ 0004-637X/746/1/85

58. W.J. Percival et al., Mon. Not. R. Astron. Soc. 401, 2148 (2010). doi:10.1111/j.1365-2966.2009.15812.x

59. F. Beutler, C. Blake, M. Colless, D.H. Jones, L. Staveley-Smith, L. Campbell, Q. Parker, W. Saunders, F. Watson, Mon. Not. R. Astron. Soc. 416, 3017 (2011). doi:10.1111/j.1365-2966.2011.19250.x 
60. C. Blake et al., Mon. Not. R. Astron. Soc. 425, 405 (2012). doi:10. 1111/j.1365-2966.2012.21473.x

61. W. Hu, N. Sugiyama, Astrophys. J. 471, 542 (1996). doi:10.1086/ 177989

62. D.J.Eisenstein, W. Hu, Astrophys. J. 496, 605 (1998). doi:10.1086/ 305424

63. J. Simon, L. Verde, R. Jimenez, Phys. Rev. D 71, 123001 (2005). doi:10.1103/PhysRevD.71.123001

64. D. Stern, R. Jimenez, L. Verde, M. Kamionkowski, S.A. Stanford, JCAP 1002, 008 (2010). doi:10.1088/1475-7516/2010/02/008

65. M. Moresco et al., JCAP 1208, 006 (2012). doi:10.1088/ $1475-7516 / 2012 / 08 / 006$

66. C. Alcock, B. Paczynski, Nature 281, 358 (1979). doi:10.1038/ $281358 \mathrm{a} 0$

67. M. Lopez-Corredoira, Astrophys. J. 781(2), 96 (2014). doi:10. 1088/0004-637X/781/2/96

68. P.M. Sutter, G. Lavaux, B.D. Wandelt, D.H. Weinberg, Astrophys. J. 761, 187 (2012). doi:10.1088/0004-637X/761/2/187

69. C. Blake et al., Mon. Not. R. Astron. Soc. 418, 1725 (2011). doi:10. 1111/j.1365-2966.2011.19606.x

70. N.P. Ross et al., Mon. Not. R. Astron. Soc. 381, 573 (2007). doi:10. 1111/j.1365-2966.2007.12289.x
71. C. Marinoni, A. Buzzi, Nature 468(7323), 539 (2010). doi:10.1038/ nature09577

72. J. da Angela, P.J. Outram, T. Shanks, Mon. Not. R. Astron. Soc. 361, 879 (2005). doi:10.1111/j.1365-2966.2005.09212.x

73. P.J. Outram, T. Shanks, B.J. Boyle, S.M. Croom, F. Hoyle, N.S. Loaring, L. Miller, R.J. Smith, Mon. Not. R. Astron. Soc. 348, 745 (2004). doi:10.1111/j.1365-2966.2004.07348.x

74. L. Anderson et al., Mon. Not. R. Astron. Soc. 427(4), 3435 (2013). doi:10.1111/j.1365-2966.2012.22066.x

75. I. Paris et al., Astron. Astrophys. 548, A66 (2012). doi:10.1051/ 0004-6361/201220142

76. D.P. Schneider et al., Astron. J. 139, 2360 (2010). doi:10.1088/ 0004-6256/139/6/2360

77. P.A.R. Ade et al., Astron. Astrophys. 594, A14 (2016). doi:10. 1051/0004-6361/201525814

78. N. Metropolis, A.W. Rosenbluth, M.N. Rosenbluth, A.H. Teller, E. Teller, J. Chem. Phys. 21, 1087 (1953). doi:10.1063/1.1699114

79. W.K. Hastings, Biometrika 57, 97 (1970). doi:10.1093/biomet/57. 1.97 